\title{
A Characterization of E-Benson Proper Efficiency via Nonlinear Scalarization in Vector Optimization
}

\author{
Ke Quan Zhao, Yuan Mei Xia, and Hui Guo \\ College of Mathematics Science, Chongqing Normal University, Chongqing 401331, China \\ Correspondence should be addressed to Ke Quan Zhao; kequanz@163.com
}

Received 22 February 2014; Accepted 14 April 2014; Published 28 April 2014

Academic Editor: Xian-Jun Long

Copyright (C) $2014 \mathrm{Ke}$ Quan Zhao et al. This is an open access article distributed under the Creative Commons Attribution License, which permits unrestricted use, distribution, and reproduction in any medium, provided the original work is properly cited.

A class of vector optimization problems is considered and a characterization of $E$-Benson proper efficiency is obtained by using a nonlinear scalarization function proposed by Göpfert et al. Some examples are given to illustrate the main results.

\section{Introduction}

It is well known that approximate solutions have been playing an important role in vector optimization theory and applications. During the recent years, there are a lot of works related to vector optimization and some concepts of approximate solutions of vector optimization problems are proposed and some characterizations of these approximate solutions are studied; see, for example, [1-3] and the references therein.

Recently, Chicoo et al. proposed the concept of $E$ efficiency by means of improvement sets in a finite dimensional Euclidean space in [4]. E-efficiency unifies some known exact and approximate solutions of vector optimization problems. Zhao and Yang proposed a unified stability result with perturbations by virtue of improvement sets under the convergence of a sequence of sets in the sense of Wijsman in [5]. Furthermore, Gutiérrez et al. generalized the concepts of improvement sets and E-efficiency to a general Hausdorff locally convex topological linear space in [6]. Zhao et al. established linear scalarization theorem and Lagrange multiplier theorem of weak $E$-efficient solutions under the nearly $E$-subconvexlikeness in [7]. Moreover, Zhao and Yang also introduced a kind of proper efficiency, named $E$-Benson proper efficiency which unifies some proper efficiency and approximate proper efficiency, and obtained some characterizations of $E$-Benson proper efficiency in terms of linear scalarization in [8].

Motivated by the works of $[8,9]$, by making use of a kind of nonlinear scalarization functions proposed by
Göpfert et al., we establish nonlinear scalarization results of $E$-Benson proper efficiency in vector optimization. We also give some examples to illustrate the main results.

\section{Preliminaries}

Let $X$ be a linear space and let $Y$ be a real Hausdorff locally convex topological linear space. For a nonempty subset $A$ in $Y$, we denote the topological interior, the topological closure, and the boundary of $A$ by int $A, \operatorname{cl} A$, and $\partial A$, respectively. The cone generated by $A$ is defined as

$$
\text { cone } A=\bigcup_{\alpha \geq 0} \alpha A \text {. }
$$

A cone $A \subset Y$ is pointed if $A \cap(-A)=\{0\}$. Let $K$ be a closed convex pointed cone in $Y$ with nonempty topological interior. For any $x, y \in Y$, we define

$$
x \leq_{K} y \Longleftrightarrow y-x \in K .
$$

In this paper, we consider the following vector optimization problem:

$$
\min _{x \in D} f(x)
$$

where $f: X \rightarrow Y$ and $\emptyset \neq D \subset X$.

Definition 1 (see $[4,6]$ ). Let $E \subset Y$. If $0 \notin E$ and $E+K=E$, then $E$ is said to be an improvement set with respect to $K$. 
Remark 2. If $E \neq \emptyset$, then, from Theorem 3.1 in [8], it is clear that int $E \neq \emptyset$. Throughout this paper, we assume that $E \neq \emptyset$.

Definition 3 (see [8]). Let $E \subset Y$ be an improvement set with respect to $K$. A feasible point $x_{0} \in D$ is said to be an $E$-Benson proper efficient solution of (VP) if

$$
\mathrm{cl}\left(\text { cone }\left(f(D)+E-f\left(x_{0}\right)\right)\right) \cap(-K)=\{0\} .
$$

We denote the set of all $E$-Benson proper efficient solutions by $x_{0} \in \operatorname{PAE}(f, E)$.

Consider the following scalar optimization problem:

$$
\min _{x \in Z} \phi(x)
$$

where $\phi: X \rightarrow \mathbb{R}, \emptyset \neq Z \subset X$. Let $\epsilon \geq 0$ and $x_{0} \in Z$. If $\phi(x) \geq \phi\left(x_{0}\right)-\epsilon$, for all $x \in Z$, then $x_{0}$ is called an $\epsilon$-minimal solution of (P). The set of all $\epsilon$-minimal solutions is denoted by $\operatorname{AMin}(\phi, \epsilon)$. Moreover, if $\phi(x)>\phi\left(x_{0}\right)-\epsilon$, for all $x \in Z$, then $x_{0}$ is called a strictly $\epsilon$-minimal solution of $(\mathrm{P})$. The set of all strictly $\epsilon$-minimal solutions is denoted by $\operatorname{SAMin}(\phi, \epsilon)$.

\section{A Characterization of $E$-Benson Proper Efficiency}

In this section, we give a characterization of $E$-Benson proper efficiency of (VP) via a kind of nonlinear scalarization function proposed by Göpfert et al.

Let $\xi_{q, E}: Y \rightarrow \mathbb{R} \cup\{ \pm \infty\}$ be defined by

$$
\xi_{q, E}(y)=\inf \{s \in \mathbb{R} \mid y \in s q-E\}, \quad y \in Y,
$$

with $\inf \emptyset=+\infty$.

Lemma 4. Let $E \subset Y$ be a closed improvement set with respect to $K$ and $q \in \operatorname{int} K$. Then $\xi_{q, E}$ is continuous and

$$
\begin{gathered}
\left\{y \in Y \mid \xi_{q, E}(y)<c\right\}=c q-\operatorname{int} E, \quad \forall c \in \mathbb{R}, \\
\left\{y \in Y \mid \xi_{q, E}(y)=c\right\}=c q-\partial E, \quad \forall c \in \mathbb{R}, \\
\xi_{q, E}(-E) \leq 0, \quad \xi_{q, E}(-\partial E)=0 .
\end{gathered}
$$

Proof. This can be easily seen from Proposition 2.3.4 and Theorem 2.3.1 in [9].

Consider the following scalar optimization problem:

$$
\min _{x \in D} \xi_{q, E}(f(x)-y), \quad\left(\mathrm{P}_{q, y}\right)
$$

where $y \in Y, q \in$ int $K$. Denote $\xi_{q, E}(f(x)-y)$ by $\left(\xi_{q, E, y} \circ\right.$ $f)(x)$, the set of $\epsilon$-minimal solutions of $\left(\mathrm{P}_{q, y}\right)$ by $\operatorname{AMin}\left(\xi_{q, E, y^{\circ}}\right.$ $f, \epsilon)$, and the set of strictly $\epsilon$-minimal solutions of $\left(\mathrm{P}_{q, y}\right)$ by $\operatorname{SAMin}\left(\xi_{q, E, y} \circ f, \epsilon\right)$.

Theorem 5. Let $E \subset Y$ be a closed improvement set with respect to $K, q \in \operatorname{int}(E \cap K)$ and $\epsilon=\inf \left\{s \in \mathbb{R}_{++} \mid s q \epsilon\right.$ $\operatorname{int}(E \cap K)\}$. Then

$$
\text { (i) } x_{0} \in \operatorname{PAE}(f, E) \Rightarrow x_{0} \in \operatorname{AMin}\left(\xi_{q, E, f\left(x_{0}\right)} \circ f, \epsilon\right) \text {; }
$$

(ii) additionally, if cone $\left(f(D)+E-f\left(x_{0}\right)\right)$ is a closed set, then

$$
x_{0} \in \operatorname{SAMin}\left(\xi_{q, E, f\left(x_{0}\right)} \circ f, \epsilon\right) \Longrightarrow x_{0} \in \operatorname{PAE}(f, E) .
$$

Proof. We first prove (i). Assume that $x_{0} \in \operatorname{PAE}(f, E)$. Then we have

$$
\mathrm{cl}\left(\text { cone }\left(f(D)+E-f\left(x_{0}\right)\right)\right) \cap(-K)=\{0\} .
$$

Therefore,

$$
\left(f(D)+E-f\left(x_{0}\right)\right) \cap(-\operatorname{int} K)=\emptyset .
$$

We can prove that

$$
\left(f\left(x_{0}\right)-\operatorname{int} E\right) \cap f(D)=\emptyset .
$$

On the contrary, there exists $\widehat{x} \in D$ such that

$$
f(\widehat{x})-f\left(x_{0}\right) \in-\operatorname{int} E .
$$

Hence, from Theorem 3.1 in [8], it follows that

$$
f(\widehat{x})-f\left(x_{0}\right) \in-E-\operatorname{int} K
$$

Therefore,

$$
f(\widehat{x})-f\left(x_{0}\right)+E \subset-\operatorname{int} K,
$$

which contradicts (8) and so (9) holds. From Lemma 4, we obtain

$$
\left\{y \in Y \mid \xi_{q, E}(y)<0\right\}=-\operatorname{int} E .
$$

From (9), we have

$$
\left(f(D)-f\left(x_{0}\right)\right) \cap(-\operatorname{int} E)=\emptyset .
$$

By using (13) and (14), we deduce that

$$
\left(f(D)-f\left(x_{0}\right)\right) \cap\left\{y \in Y \mid \xi_{q, E}(y)<0\right\}=\emptyset .
$$

Thus,

$$
\left(\xi_{q, E, f\left(x_{0}\right)} \circ f\right)(x)=\xi_{q, E}\left(f(x)-f\left(x_{0}\right)\right) \geq 0, \quad \forall x \in D .
$$

In addition, since $\left\{s \in \mathbb{R}_{++} \mid s q \in \operatorname{int}(E \cap K)\right\} \subset\{s \in \mathbb{R} \mid s q \in$ $E\}$,

$$
\left(\xi_{q, E, f\left(x_{0}\right)} \circ f\right)\left(x_{0}\right)=\xi_{q, E}(0)=\inf \{s \in \mathbb{R} \mid s q \in E\} \leq \epsilon .
$$

It follows from (16) that

$$
\left(\xi_{q, E, f\left(x_{0}\right)} \circ f\right)(x) \geq\left(\xi_{q, E, f\left(x_{0}\right)} \circ f\right)\left(x_{0}\right)-\epsilon .
$$

Therefore, $x_{0} \in \operatorname{AMin}\left(\xi_{q, E, f\left(x_{0}\right)} \circ f, \epsilon\right)$.

Next, we prove (ii). Suppose that $x_{0} \in \operatorname{SAMin}\left(\xi_{q, E, f\left(x_{0}\right)}{ }^{\circ}\right.$ $f, \epsilon)$ and $x_{0} \notin \operatorname{PAE}(f, E)$. Since cone $\left(f(D)+E-f\left(x_{0}\right)\right)$ is 
a closed set, there exist $0 \neq d \in-K, \lambda>0, \widehat{x} \in D$, and $\widehat{e} \in E$ such that

$$
d=\lambda\left(f(\widehat{x})-f\left(x_{0}\right)+\widehat{e}\right) .
$$

Since $K$ is a cone,

$$
f(\widehat{x})-f\left(x_{0}\right)+\widehat{e} \in-K .
$$

Therefore, we can obtain that

$$
f(\widehat{x})-f\left(x_{0}\right) \in-\widehat{e}-K \subset-E-K=-E .
$$

Moreover, by Lemma 4 , we have, for every $c \in \mathbb{R}$,

$$
\begin{aligned}
c q+f(\widehat{x})-f\left(x_{0}\right) & \in c q-E \\
& =c q-c l E \\
& =\left\{y \in Y \mid \xi_{q, E}(y) \leq c\right\} ;
\end{aligned}
$$

that is,

$$
\xi_{q, E}\left(c q+f(\widehat{x})-f\left(x_{0}\right)\right) \leq c .
$$

Let $c=0$ in (23); then, we have

$$
\xi_{q, E}\left(f(\widehat{x})-f\left(x_{0}\right)\right) \leq 0 .
$$

On the other hand, from $x_{0} \in \operatorname{SAMin}\left(\xi_{q, E, f\left(x_{0}\right)} \circ f, \epsilon\right)$, it follows that

$$
\begin{aligned}
\xi_{q, E}\left(f(\widehat{x})-f\left(x_{0}\right)\right) & >\xi_{q, E}\left(f\left(x_{0}\right)-f\left(x_{0}\right)\right)-\epsilon \\
& =\xi_{q, E}(0)-\epsilon .
\end{aligned}
$$

In the following, we prove

$$
\xi_{q, E}(0)=\epsilon .
$$

We first point out that, for any $s \leq 0, s q \notin E$. It is obvious that $0 \notin E$ when $s=0$. Assume that there exists $\widehat{s}<0$ such that $\widehat{s} q \in E$. Since $q \in \operatorname{int}(E \cap K) \subset K$ and $-\widehat{s} q \in K$, we have

$$
0=\widehat{s} q-\widehat{s} q \in E+K=E,
$$

which contradicts the fact that $E$ is an improvement set with respect to $K$. Hence,

$$
\begin{aligned}
\xi_{q, E}(0) & =\inf \{s \in \mathbb{R} \mid 0 \in s q-E\} \\
& =\inf \left\{s \in \mathbb{R}_{++} \mid s q \in E\right\} .
\end{aligned}
$$

Moreover, since $q \in \operatorname{int}(E \cap K) \subset K$, we have, for any $s \in \mathbb{R}_{++}$, $s q \in K$. It follows from (28) that

$$
\xi_{q, E}(0)=\inf \left\{s \in \mathbb{R}_{++} \mid s q \in E \cap K\right\} .
$$

Hence (26) holds and thus, by (25), we obtain $\xi_{q, E}(f(\widehat{x})-$ $\left.f\left(x_{0}\right)\right)>0$, which contradicts (24) and so $x_{0} \in$ $\operatorname{PAE}(f, E)$.

Remark 6. $x_{0} \in \operatorname{PAE}(f, E)$ does not imply $x_{0} \in$ $\operatorname{SAMin}\left(\xi_{q, E, f\left(x_{0}\right)} \circ f, \epsilon\right)$.
Example 7. Let $X=Y=\mathbb{R}^{2}, K=\mathbb{R}_{+}^{2}, f(x)=x$, and

$$
\begin{aligned}
& E=\left\{\left(x_{1}, x_{2}\right) \mid x_{1}+x_{2} \geq 1, x_{1} \geq 0, x_{2} \geq 0\right\}, \\
& D=\left\{\left(x_{1}, x_{2}\right) \mid x_{1}-x_{2}=0,-\frac{1}{2} \leq x_{1} \leq 0\right\} .
\end{aligned}
$$

Clearly, $K$ is a closed convex cone and $E$ is a closed improvement set with respect to $K$. Let $x_{0}=(0,0) \in D$ and $q=(1,1) \in \operatorname{int}(E \cap K)$. Then $\epsilon=1 / 2$ since

$$
\begin{aligned}
& \operatorname{cl}\left(\text { cone }\left(f(D)+E-f\left(x_{0}\right)\right)\right) \cap(-K) \\
& \quad=\left\{\left(x_{1}, x_{2}\right) \mid x_{1}+x_{2} \geq 0\right\} \cap\left(-\mathbb{R}_{+}^{2}\right)=\{(0,0)\} .
\end{aligned}
$$

Hence

$$
x_{0} \in \operatorname{PAE}(f, E) .
$$

For any $x \in D$,

$$
\begin{aligned}
\xi_{q, E}\left(f(x)-f\left(x_{0}\right)\right) & =\xi_{q, E}(f(x)) \\
& =\inf \{s \in \mathbb{R} \mid f(x) \in s q-E\} \\
& \geq 0=\frac{1}{2}-\frac{1}{2} \\
& =\xi_{q, E}(0)-\epsilon .
\end{aligned}
$$

Therefore,

$$
x_{0} \in \operatorname{AMin}\left(\xi_{q, E, f\left(x_{0}\right)} \circ f, \epsilon\right) .
$$

However, there exists $\widehat{x}=(-1 / 2,-1 / 2) \in D$ such that

$$
\begin{aligned}
\xi_{q, E}\left(f(\widehat{x})-f\left(x_{0}\right)\right) & =\xi_{q, E}(f(\widehat{x})) \\
& =\inf \{s \in \mathbb{R} \mid f(\widehat{x}) \in s q-E\} \\
& =0=\frac{1}{2}-\frac{1}{2} \\
& =\xi_{q, E}(0)-\epsilon .
\end{aligned}
$$

Hence

$$
x_{0} \notin \operatorname{SAMin}\left(\xi_{q, E, f\left(x_{0}\right)} \circ f, \epsilon\right) .
$$

Remark 8. Theorem 5(ii) may not be true if the closedness of cone $\left(f(D)+E-f\left(x_{0}\right)\right)$ is removed and the following example can illustrate it.

Example 9. Let $X=Y=\mathbb{R}^{2}, K=\mathbb{R}_{+}^{2}, f(x)=x$, and

$$
\begin{gathered}
E=\left\{\left(x_{1}, x_{2}\right) \mid x_{1}+x_{2} \geq 1, x_{1} \geq 0, x_{2} \geq \frac{1}{2}\right\}, \\
D=\left\{\left(x_{1}, x_{2}\right) \mid x_{1} \leq 0, x_{2}=0\right\} .
\end{gathered}
$$

Clearly, $K$ is a closed convex cone and $E$ is a closed improvement set with respect to $K$. Let $x_{0}=(0,0) \in D$ and $q=(1,1) \in \operatorname{int}(E \cap K)$. Then $\epsilon=1 / 2$ and

$$
\begin{aligned}
\text { cone } & \left(f(D)+E-f\left(x_{0}\right)\right) \\
& =\left\{\left(x_{1}, x_{2}\right) \mid x_{1} \in \mathbb{R}, x_{2}>0\right\} \cup\{(0,0)\}
\end{aligned}
$$


is not a closed set, since for any $x \in D$

$$
\begin{aligned}
\xi_{q, E}\left(f(x)-f\left(x_{0}\right)\right) & =\xi_{q, E}(f(x)) \\
& =\inf \{s \in \mathbb{R} \mid f(x) \in s q-E\} \\
& =\frac{1}{2}>\frac{1}{2}-\frac{1}{2} \\
& =\xi_{q, E}(0)-\epsilon .
\end{aligned}
$$

Therefore,

$$
x_{0} \in \operatorname{SAMin}\left(\xi_{q, E, f\left(x_{0}\right)} \circ f, \epsilon\right) .
$$

However,

$$
\begin{aligned}
& \operatorname{cl}\left(\text { cone }\left(f(D)+E-f\left(x_{0}\right)\right)\right) \cap(-K) \\
& =\left\{\left(x_{1}, x_{2}\right) \mid x_{1} \in \mathbb{R}, x_{2} \geq 0\right\} \cap\left(-\mathbb{R}_{+}^{2}\right) \\
& =\left\{\left(x_{1}, x_{2}\right) \mid x_{1} \leq 0, x_{2}=0\right\} \neq\{(0,0)\} \text {. }
\end{aligned}
$$

Therefore,

$$
x_{0} \notin \operatorname{PAE}(f, E) .
$$

Remark 10. Theorem 5(ii) may not be true if $x_{0} \in$ $\operatorname{SAMin}\left(\xi_{q, E, f\left(x_{0}\right)} \circ f, \epsilon\right)$ is replaced by $x_{0} \in \operatorname{AMin}\left(\xi_{q, E, f\left(x_{0}\right)} \circ\right.$ $f, \epsilon)$ and the following example can illustrate it.

Example 11. Let $X=Y=\mathbb{R}^{2}, K=\mathbb{R}_{+}^{2}, f(x)=x$, and

$$
\begin{aligned}
E= & \left\{\left(x_{1}, x_{2}\right) \mid x_{1}+x_{2} \geq 1, x_{1} \geq \frac{1}{2}, x_{2} \geq 0\right\} \\
& \cup\left\{\left(x_{1}, x_{2}\right) \mid x_{1} \leq \frac{1}{2}, x_{2} \geq \frac{1}{2}\right\}, \\
D= & \left\{\left(x_{1}, x_{2}\right) \mid x_{1}-x_{2}=0,-\frac{1}{2} \leq x_{1} \leq 0\right\} .
\end{aligned}
$$

Clearly, $K$ is a closed convex cone and $E$ is a closed improvement set with respect to $K$. Let $x_{0}=(0,0) \in D$ and $q=(1,1) \epsilon \operatorname{int}(E \cap K)$. Then $\epsilon=1 / 2$ and

$$
\begin{aligned}
\text { cone } & \left(f(D)+E-f\left(x_{0}\right)\right) \\
= & \left\{\left(x_{1}, x_{2}\right) \mid x_{1} \in \mathbb{R}, x_{2} \geq 0\right\} \\
& \cup\left\{\left(x_{1}, x_{2}\right) \mid x_{1}+x_{2} \geq 0, x_{1} \geq 0, x_{2} \leq 0\right\}
\end{aligned}
$$

is a closed set, since for any $x \in D$

$$
\begin{aligned}
\xi_{q, E}\left(f(x)-f\left(x_{0}\right)\right) & =\xi_{q, E}(f(x)) \\
& =\inf \{s \in \mathbb{R} \mid f(x) \in s q-E\} \\
& \geq 0=\frac{1}{2}-\frac{1}{2} \\
& =\xi_{q, E}(0)-\epsilon .
\end{aligned}
$$

Therefore,

$$
x_{0} \in \operatorname{AMin}\left(\xi_{q, E, f\left(x_{0}\right)} \circ f, \epsilon\right)
$$

However, there exists $\widehat{x}=(-1 / 2,-1 / 2) \in D$ such that

$$
\begin{aligned}
\xi_{q, E}\left(f(\widehat{x})-f\left(x_{0}\right)\right) & =\xi_{q, E}(f(\widehat{x})) \\
& =\inf \{s \in \mathbb{R} \mid f(\widehat{x}) \in s q-E\} \\
& =0=\frac{1}{2}-\frac{1}{2} \\
& =\xi_{q, E}(0)-\epsilon .
\end{aligned}
$$

Hence,

$$
x_{0} \notin \operatorname{SAMin}\left(\xi_{q, E, f\left(x_{0}\right)} \circ f, \epsilon\right) .
$$

Moreover,

$$
\begin{aligned}
\operatorname{cl}(\text { cone } & \left.\left(f(D)+E-f\left(x_{0}\right)\right)\right) \cap(-K) \\
= & \left\{\left(x_{1}, x_{2}\right) \mid x_{1} \in \mathbb{R}, x_{2} \geq 0\right\} \\
& \cup\left\{\left(x_{1}, x_{2}\right) \mid x_{1}+x_{2} \geq 0, x_{1} \geq 0, x_{2} \leq 0\right\} \cap\left(-\mathbb{R}_{+}^{2}\right) \\
= & \left\{\left(x_{1}, x_{2}\right) \mid x_{1} \leq 0, x_{2}=0\right\} \neq\{(0,0)\} .
\end{aligned}
$$

Therefore,

$$
x_{0} \notin \operatorname{PAE}(f, E) \text {. }
$$

\section{Conflict of Interests}

The authors declare that there is no conflict of interests regarding the publication of this paper.

\section{Acknowledgments}

This work is partially supported by the National Natural Science Foundation of China (Grant nos. 11301574, 11271391, and 11171363), the Natural Science Foundation Project of Chongqing (Grant no. CSTC2012jjA00002), and the Research Fund for the Doctoral Program of Chongqing Normal University (13XLB029).

\section{References}

[1] C. Gutiérrez, B. Jiménez, and V. Novo, "A unified approach and optimality conditions for approximate solutions of vector optimization problems," SIAM Journal on Optimization, vol. 17, no. 3, pp. 688-710, 2006.

[2] Y. Gao, X. Yang, and K. L. Teo, "Optimality conditions for approximate solutions of vector optimization problems," Journal of Industrial and Management Optimization, vol. 7, no. 2, pp. 483-496, 2011.

[3] F. Flores-Bazán and E. Hernández, "A unified vector optimization problem: complete scalarizations and applications," Optimization, vol. 60, no. 12, pp. 1399-1419, 2011.

[4] M. Chicco, F. Mignanego, L. Pusillo, and S. Tijs, "Vector optimization problem via improvement sets," Journal of Optimization Theory and Applications, vol. 150, no. 3, pp. 516-529, 2011. 
[5] K. Q. Zhao and X. M. Yang, "A unified stability result with perturbations in vector optimization," Optimization Letters, vol. 7, no. 8, pp. 1913-1919, 2013.

[6] C. Gutiérrez, B. Jiménez, and V. Novo, "Improvement sets and vector optimization," European Journal of Operational Research, vol. 223, no. 2, pp. 304-311, 2012.

[7] K. Q. Zhao, X. M. Yang, and J. W. Peng, "Weak E-optimal solution in vector optimization," Taiwanese Journal of Mathematics, vol. 17, no. 4, pp. 1287-1302, 2013.

[8] K. Q. Zhao and X. M. Yang, "E-Benson proper efficiency in vector optimization,” Optimization, 2013.

[9] A. Göpfert, C. Tammer, H. Riahi, and C. Zălinescu, Variational Methods in Partially Ordered Spaces, Springer, New York, NY, USA, 2003. 


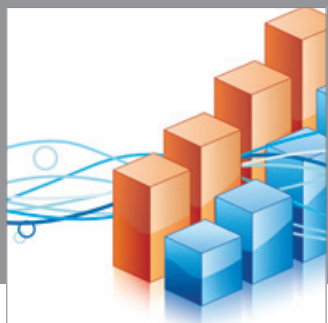

Advances in

Operations Research

mansans

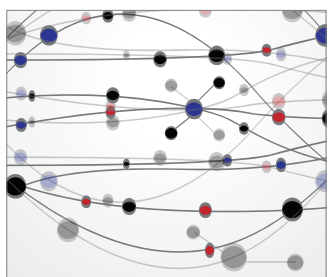

The Scientific World Journal
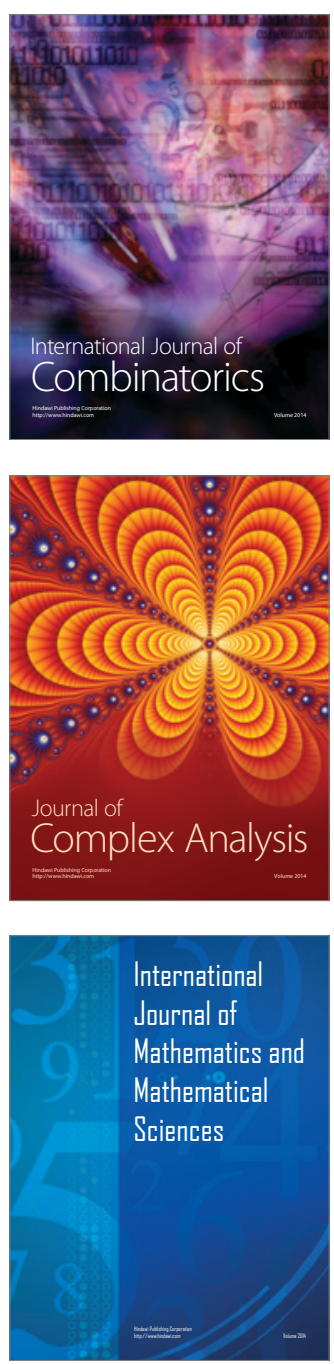
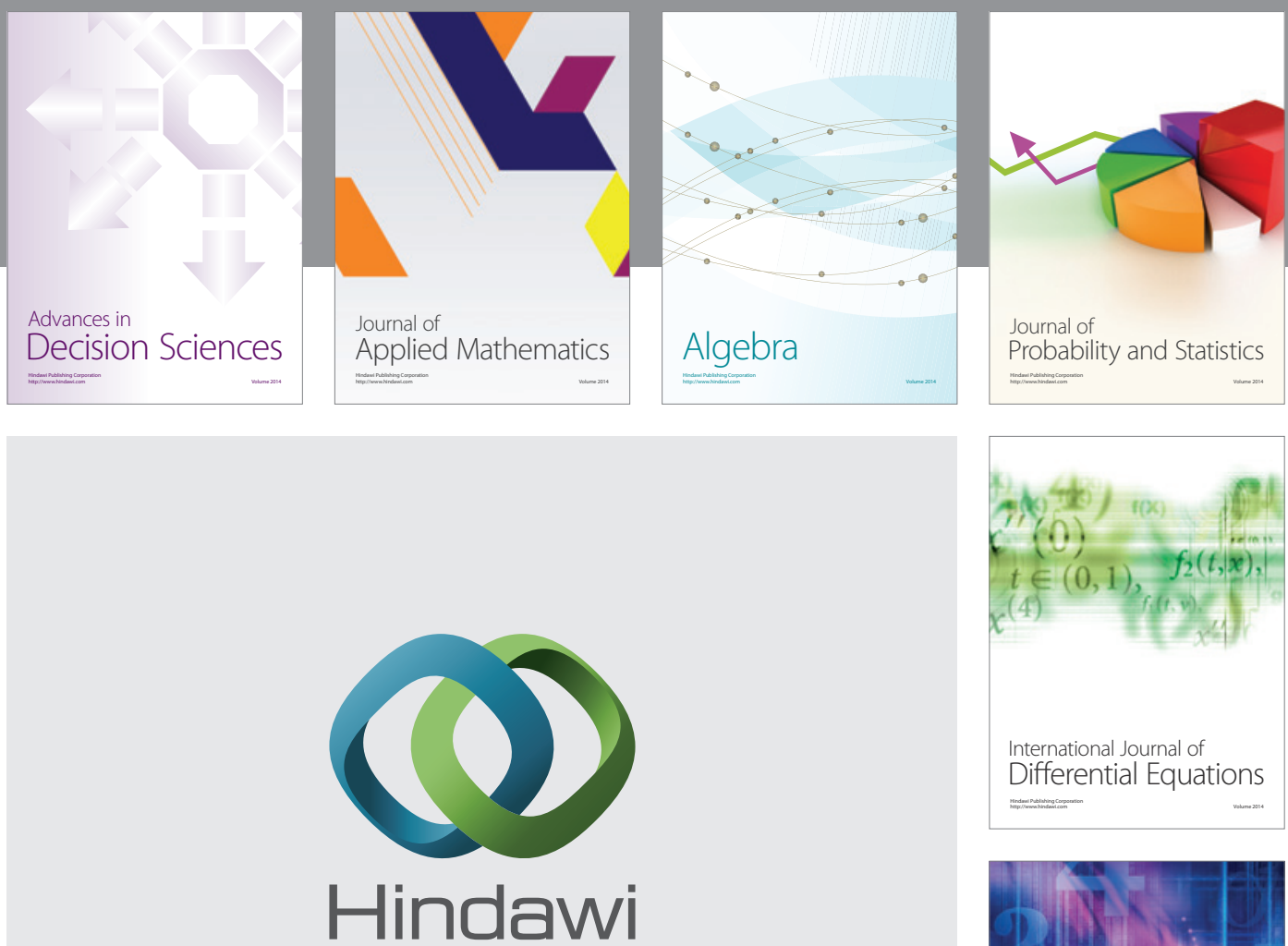

Submit your manuscripts at http://www.hindawi.com
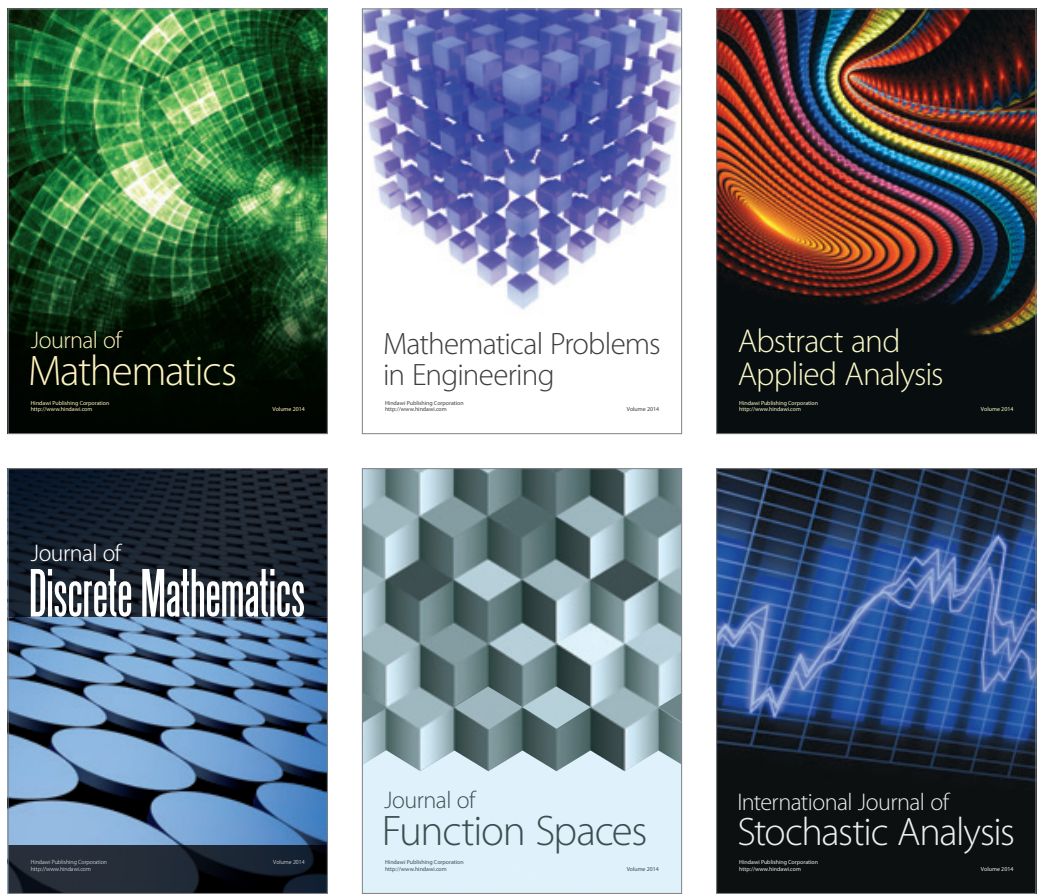

Journal of

Function Spaces

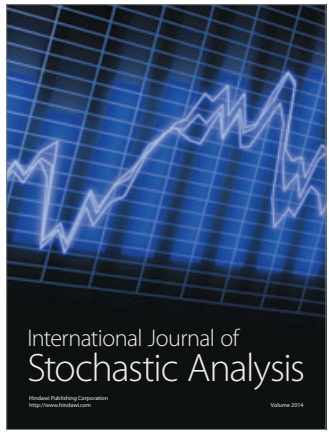

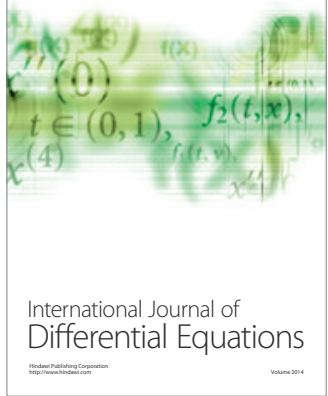
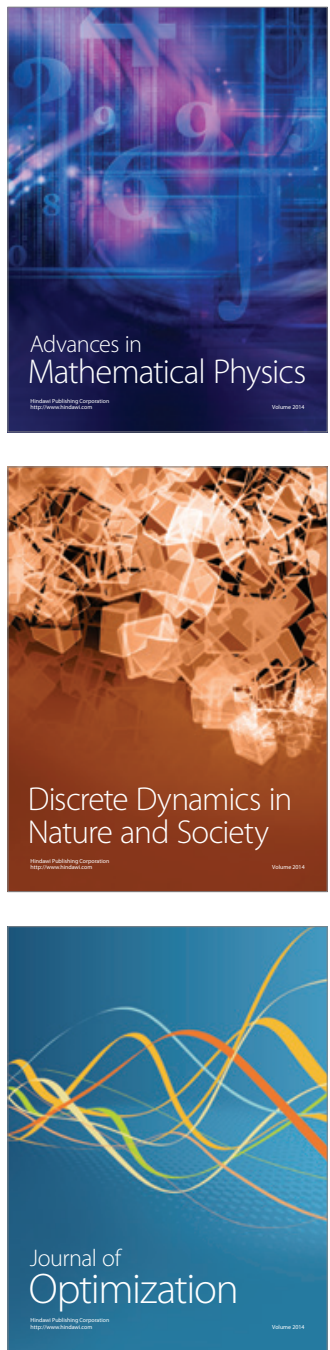